\title{
GW23-e2379 MIR-27B EXPRESSION AND SIGNIFICANCE OF PULMONARY HYPERTENSION IN CONGENITAL HEART DISEASE
}

doi:10.1136/heartjnl-2012-302920p.4

Zhicheng Wu, Chunfa Xie, Zixiang Zhang. First Affiliated Hospital of Gannan Medical College

Objectives To investigate the clinical significance of blood brain natriuretic peptide (BNP) testing in patients with acute dyspnoea due to differential due to cardiac and pulmonary.

Methods The selected object: Select January 2011 to January 2012, Gannan Medical College First Affiliated Hospital, Department of Cardiothoracic Surgery, Department of Respiratory Medicine, Department of Cardiology, emergency inpatient treatment of patients with acute dyspnoea included in the study. Trauma, cardiac tamponade, acute coronary syndrome, congenital heart disease, renal insufficiency diseases were excluded. Selected 231 cases of difficulty in breathing patients in our hospital, 105 cases of 126 cases of male, female, cardiogenic group of 96 cases, 145 cases of pulmonary group. Methods: mining outer peripheral venous blood $1 \mathrm{ml}$ EDTA, the application of Bayer company ACS: 180 immunoluminometric quantitative testing equipment, the use of the Bayer BNP reagents and double-antibody sandwich immunofluorescence assay plasma BNP concentrations, the normal value is 0-100 ng/l, respectively. The application GEVivid5 systemic digital colour Doppler ultrasound conventional echocardiography, measured and recorded LVED and LVEF.

Results (1) Cardiac group: The average level of plasma BNP (604.78 $\pm 137.70) \mathrm{ng} / \mathrm{l}$, pulmonary group: BNP average (43.56 \pm 32.73$) \mathrm{ng} / \mathrm{l}$, significantly lower than the cardiac group $(p<0.01)$. Plasma BNP concentrations of heart failure severity increased can be drawn from the cardiac group, of which 28 cases of NYHA grade II, III grade 30 cases, 23 cases of grade IV, BNP levels (246.56 \pm 883.42 ) ng/l, (568.23 \pm 153.68$) \mathrm{ng} / \mathrm{l}$, and (1123.83 \pm 186.56$) \mathrm{ng} / \mathrm{l}$. (2) Two groups of patients with plasma BNP levels, LVED, the comparison of LVEF, heart failure patients with BNP levels and LVED was positively correlated $(r=0.56, p<0.01)$, and LVEF was negatively correlated $(\mathrm{r}=-0.53, \mathrm{p}<0.01)$. 34 cases of NYHA grade II, 36 cases of grade III, 26 cases of grade IV, the LVED value, respectively (56.4 $\pm 5.8) \mathrm{mm}(61.7 \pm 6.5) \mathrm{mm}(65.4 \pm 3.7) \mathrm{mm}$. LVEF $(48 \pm 9) \%$, (40 $\pm 8) \%$, $(30 \pm 6) \%$.

Conclusions Compared with cardiac dyspnoea group and pulmonary dyspnoea group, BNP levels were significantly increased, and is proportional to the BNP and the severity of heart failure, heart failure, BNP levels were positively correlated with LVED was negatively correlated with LVEFprompt the release of BNP directly related to ventricular pressure load over, with the rise of ventricular pressure overload, ventricular myocytes increased secretion, and to determine the severity and changes in patients with heart failure by BNP. With the advent of the BNP rapid diagnostic tests, clinical detection of BNP levels has become more sophisticated, timely detection of clinical bedside quickly distinguish between dyspnoea, 
early diagnosis and rational treatment of patients with breathing difficulties to provide excellent technical support. 